\title{
A RAPID, SENSITIVE AND VALIDATED ULTRA PERFORMANCE LIQUID CHROMATOGRAPHY AND TANDEM MASS SPECTROMETRY METHOD FOR DETERMINATION OF RIFAMPICIN IN RAT PLASMA: APPLICATION TO PHARMACOKINETIC STUDY
}

\author{
ASLAM BURHAN ${ }^{*}$, BHAVIN VYAS ${ }^{1}$ \\ 1Department of Pharmacology, Maliba Pharmacy College, UKA Tarsadia University, Maliba Campus, Gopal Vidyanagar, Bardoli-Mahuva \\ Road, Tarsadi, Taluka Mahuva, Surat, Gujarat 394350, India \\ Email: burhanaslam@gmail.com \\ Received: 25 Oct 2016 Revised and Accepted: 21 Dec 2016
}

\section{ABSTRACT}

Objective: To develop and validate simple, sensitive and selective ultra-performance liquid chromatography and tandem mass spectrometry (UPLC-MS/MS) method for quantification of rifampicin (RIF) in rat plasma and its application to pharmacokinetics study.

Methods: Precipitation method was used for the extraction of plasma samples, an aliquot of $25 \mu$ l plasma samples was extracted using acetonitrile precipitation technique. Chromatographic separation was performed usingWaters Acquity ${ }^{\mathrm{TM} U P L C}$ columns, BEH C18 (50 mm $\left.\times 2.1 \mathrm{~mm}, 1.7 \mu \mathrm{m}\right)$ by a gradient mixture of acetonitrile and water (both containing $0.1 \%$ formic acid) as a mobile phase at the flow rate of $0.7 \mathrm{ml} / \mathrm{min}$.The analyte was protonated in the positive ESI (electrospray ionization interface) and detected in MRM (multiple reactions monitoring) modes using the transition $\mathrm{m} / \mathrm{z}$ 308.60-455.30.

Results: The method had a short chromatography run time of $1.8 \mathrm{~min}$ with improved sensitivity over existing methods. Calibration curves been linear over the wide range of 1.97-5047.00 ng/ml. The between and within-batch precision and accuracy of the method was determined by using 4 quality control samples; the highest \%CV observed was10.11. The mean recovery values are 74.26, 82.77 and 101.73 at low, medium and highquality control levels; respectively.

Conclusion: It was concluded that the developed and validated UPLC-MS/MS method was sensitive,specific, precise, linear, and rapid. Therefore, the method can be used for quantification of RIFin rat plasma with various advantages over the reported methods. RIF is widely recommended by US-FDAguidance for industry on drug interaction studies and the developed method can be used to explore drug interaction studies in drug discovery and development.

Keywords: Rifampicin, Quantification,Pharmacokinetics, Drug interaction, UPLC-MS/MS

(C) 2017 The Authors. Published by Innovare Academic Sciences Pvt Ltd. This is an open access article under the CC BY license (http://creativecommons.org/licenses/by/4. 0/) DOI: http://dx.doi.org/10.22159/ijpps.2017v9i2.15865

\section{INTRODUCTION}

RIF is a major antibacterial drug used for the treatment of tuberculosis, leprosy, some types of osteomyelitis and endocarditis, etc. RIF inhibits DNA-dependent RNA polymerase in bacterial cells and therefore evaluation of plasma concentration is of crucial importance. RIF is a semisynthetic derivative of a complex macrocyclic antibiotic having bactericidal activity and extensively used for the treatment of tuberculosis together with isoniazid, ethambutol and pyrazinamide [1]. Apart from this RIF is widely used for many drug-drug interaction (DDI) studies as an established inducer of multiple CYP enzymes (CYP2C8, 2C9, 2C19, 3A4; reported fold induction in enzyme activities are 2-4, 3.7, 20 and 4-31, respectively), transporters, and an inhibitor of the uptake transporter OATP1B1 and may inhibit the uptake of investigational drug that is a substrate of OATP1B1 [2-4]. Many drugs undergo metabolism and biliary excretion after sinusoidal uptake into the liver and hepatic uptake processes, which play an important role in the hepatic clearance of drugs [5].

To evaluate drug-herb interaction (DHI) studies using RIF, where an isolated active herbal constituent is not available and most of the marketed herbal products were available as amixture or aqueous extract in powder form and evaluating DHI studies in such cases is a very difficult task because no isolated fraction is available for bioanalytical quantification. In such scenario, the only option remained is to plan the drug-herb interaction studies using RIF in vivo pharmacokinetics studies in animals and thereby quantification of RIF from plasma samples and the outcomes of the studies will be correlated with CYP inhibition/induction potential of concurrently administered herb drugs on the pharmacokinetics of RIF.As reported, CYP3A4 is the most abundant cytochrome P450 enzyme expressed in the liver and small intestine and plays a crucial role in metabolism and detoxification of xenobiotics and endobiotics. CYP3A4 has very broad substrate specificity and metabolizes over $50 \%$ of clinical drugs. Some important CYP3A4 interactions are due to intestinal rather hepatic enzyme inhibition. Some potent inhibitors of CYP3A4 include clarithromycin, erythromycin, diltiazem, itraconazole, ketoconazole, ritonavir, verapamil, goldenseal and grapefruit. Inducers of CYP3A4 include phenobarbital, phenytoin, RIF, St. John's Wort and glucocorticoids. The expression levels of CYP3A4 vary widely among individual [6,7]

To summarize the objective ofDHIand DDI studies for a new drug is to determine A) whether any interactions are sufficiently large to necessitate a dosage adjustment of the drug itself or of the drugs with which it might be used B) whether any interactions calls for additional therapeutic monitoring, or C) whether any contra-indication to concomitant use when lesser measures cannot mitigate the risk [8]. Now, considering several analytical methods available for determination of RIF in biological fluids and pharmaceutical dosage forms, including high-performance thin layer chromatography (HPTLC), high-performance liquid chromatography (HPLC), UPLC, LC-MS/MS and matrix-assisted laser desorption/ionization with a time of flight (MALDI-TOF). But, none of the methods is predominant and having various drawbacks viz less sensitivity, complex extraction procedure, and large sample size and unsatisfactory quantitative analysis [9-27].

Thus considering the limitations of the various existing methods described above, the present study was performed with the aim to develop and validate a simple, sensitive, accurate and rapid UPLC- 
MS/MS method for the determination of RIF in rat plasma and its pharmacokinetics application in DHI studies. Recently there were no reports available predominantly with high sensitivity and advantageous short run time for RIF quantification in rat plasma samples.

\section{MATERIALS AND METHODS}

\section{Chemicals and reagents}

Rifampicin, albendazole and $\mathrm{K}_{2}$ EDTA, formic acid, were obtained from Sigma-Aldrich (Bangalore, India). Acetonitrile from J. T. Baker (Mumbai, India). All the chemicals used in this study were of analytical grade.

\section{Animal husbandry and handling}

All animal studies had the approval of the institutional animal ethics committee (IAEC)as per approval number MPC/IAEC/02/2015 of "UKA Tarsadia University" (animal house facility) and were in accordance with the guidelines of the CPCSEA, Government of India. Animals were acclimatized in study rooms for at least three days prior to dosing. Rats were housed in polypropylene cages ( 1 animals per cage, marked for identification) maintained in controlled environmental conditions $\left(22 \pm 3{ }^{\circ} \mathrm{C}\right.$; $40-70 \% \mathrm{RH} ; 10-15$ fresh air change cycles/h) with $12 \mathrm{~h}$ light and dark cycles. Rats were given rodent pellet diet, and UV treated filtered water ad libitum. Rat blood samples were collected from retro-orbital plexus using a serial sampling design.

\section{Instrumentation}

\section{Stock solution and calibration standards}

A standard stock solution of RIF and albendazole were prepared with a final concentration of $1.03 \mathrm{mg} / \mathrm{ml}$ and $2.10 \mathrm{mg} / \mathrm{ml}$, respectively. These solutions were stored at $2-8{ }^{\circ} \mathrm{C}$ until use. The analytical standard for RIF in acetonitrile over a concentration range of $1.97 \mathrm{ng} / \mathrm{ml}$ to $5047.00 \mathrm{ng} / \mathrm{ml}$ by serial dilution method and same concentration range for calibration curve were also prepared in blank rat plasma. Quality control (QC) samples at four different concentration levels for RIFas the lower limit of quantification (LLOQQC), low QC (LQC), medium QC (MQC) and high QC (HQC), respectively were prepared in three sets independent of the calibration standards. During analysis, these QC samples were spaced after every six to seven unknown samples.

\section{Sample preparation}

An aliquot of $25 \mu \mathrm{l}$ study sample or spiked calibration standard/QCs was added to individual pre-labeled microcentrifuge tubes followed by $25 \mu \mathrm{l}$ of IS prepared in water (albendazole, $500 \mathrm{ng} / \mathrm{ml}$ ) was added except for blank rat plasma, where $25 \mu$ l of water was added. Samples were vortexed, followed by $100 \mu \mathrm{l}$ of acetonitrile was added and samples were vortexed for another 5 min.After centrifugation of the samples at $4000 \mathrm{rpm}$ for $10 \mathrm{~min}$ at $4^{\circ} \mathrm{C}$, the supernatant was collected and $5 \mu \mathrm{l}$ was injected onto the UPLC-MS/MS system.

\section{Chromatographic condition}

A Waters Acquity ${ }^{\mathrm{TM}}$ UPLC (USA) consisting of flow control valve, vacuum degasser operated in a gradient mode to deliver the mobile phase at a flow rate of $0.7 \mathrm{ml} / \mathrm{min}$. The chromatographic system consisted of BEH C ${ }^{18}$ column $(50 \mathrm{~mm} \times 2.1 \mathrm{~mm}, 1.7 \mu \mathrm{m})$ (Waters, USA) and mobile phase consists of $0.1 \%$ Formic acid $v / v$ in acetonitrile (A),0.1\% Formic acid v/v in water (B). A gradient UPLC method with1.8 min run time was employed for analysis. The mobile phase comprised $0.1 \%$ Formic acid v/v/in acetonitrile and $0.1 \%$ Formic acid v/v in water, the flow rate was $0.7 \mathrm{ml} / \mathrm{min}$. Separation was achieved using BEH C18 column $(1.7 \mu \mathrm{m}, 2.1 \mathrm{~mm}$ x $50 \mathrm{~mm}$, $3 / \mathrm{pkg}$ ), Waters (USA), maintained at $45^{\circ} \mathrm{C}$ employing an injection volume of $5 \mu \mathrm{l}$ for in vivo rat samples.

\section{Mass spectrometric condition}

Mass spectrometric detection was performed on AB SCIEX-4000 Triple Quadrupole MS/MS mass spectrometer equipped with electron spray ionisation (ESI) source, the multiple reaction monitoring (MRM) modes was used for data acquisition with analyst
1.6.2 software. Peak integration and calibration were carried out by using analyst 1.6.2 software. MS and MS/MS condition for pure standards of RIF and IS albendazole were optimised by continuous infusion at $5 \mu \mathrm{l} / \mathrm{min}$ using syringe pump. The transitions monitored were $\mathrm{m} / \mathrm{z} 823.8(\mathrm{~m} / \mathrm{z})>151.2$ and $266.1>234.0$ for components RIF and IS albendazole, respectively. All analyzes were carried out in positive ion ESI with spray voltage set at $5500 \mathrm{~V}$. The heated ESI temperature was set $550{ }^{\circ} \mathrm{C}$. Nitrogen ion source gas GS1 and GS2 set at 40 and 60 psi, respectively. The collision assisted dissociation (CAD) was used at pressure 6 psi. Total run time for UPLC-MS/MS analysis was $1.8 \mathrm{~min}$.

\section{Application to pharmacokinetic study}

The method was successfully applied to evaluate the plasma concentration versus time profile of RIFin rat plasma following oral administration at $50 \mathrm{mg} / \mathrm{kg}$ dose of RIF.The oral dose volume was $5 \mathrm{ml} / \mathrm{kg}$ and dosing was performed using oral gauge needle. The formulation was prepared freshly on the day of dosing. Studies were performed in healthy male Wistar rat (220260 g). A serial sampling design was used ( $n=3$ per time point). Approximately, $60 \mu \mathrm{l}$ of blood samples was collected (K $\mathrm{K}_{2}$ EDTA anticoagulant, $20 \mu \mathrm{l}$ of $\mathrm{K}_{2}$ EDTA solution $/ \mathrm{ml}$ of blood, $200 \mathrm{mmol}$ ) at $0.25,0.5,1,2,4,8$ and $24 \mathrm{~h}$ postdose. Plasma samples were separated by centrifugation of whole blood and stored below$70 \pm 10{ }^{\circ} \mathrm{C}$ until bio-analysis.

Pharmacokinetic parameters were calculated using noncompartmental analysis tool of WinNonlin ${ }^{\circledR}$ software (Version 6.3). The area under the concentration-time curve ( $\mathrm{AUC}_{\text {last }}$ and $\mathrm{AUC}_{\mathrm{inf}}$ ) was calculated by linear trapezoidal rule. The peak concentration $\left(\mathrm{C}_{\max }\right)$ and time for the peak concentration $\left(\mathrm{T}_{\max }\right)$ were the observed values. The elimination rate constant value $\left(\mathrm{k}_{\mathrm{el}}\right)$ was obtained by linear regression of the log-linear terminal phase of the concentration-time profile using at least 3 non-zero declining concentrations in terminal phase with a correlation coefficient of $>0.8$.

\section{RESULTS AND DISCUSSION}

\section{Mass spectrometry}

In order to find most, sensitive ionisation mode for the components studied ESI positive ion mode and ESI negative ion model tested with the various combination of mobile phase, i.e., methanol, acetonitrile and water/ammonium acetate buffer $(2 \mathrm{mmol}) /$ formic acid $(0.1 \%)$ in positive and negative ionization mode. It was observed that the signal intensity for $[\mathrm{M}+\mathrm{H}]+$ ions in ESI positive ion mode were 5-15-fold higher for RIFusing $0.1 \%$ Formic acid v/v in acetonitrile and $0.1 \%$ Formic acid $v / v$ in water, versus experiments run with ESI negative ion mode.

The protonated molecular ion of $[\mathrm{M}+\mathrm{H}]^{+}, m / z 823.8$ were obtained for RIF and no significant solvent adduct ions or fragment ions were observed in the full scan spectra of RIF and albendazole (IS).Thus, it was decided to utilise positive ion mode for detection and quantification of $[\mathrm{M}+\mathrm{H}]^{+}$ions and representative chromatogram showed in the fig. 1 and 2.

\section{Liquid chromatography}

Acetonitrile rather than methanol was chosen as an organic modifier because of its better peak shape. A gradient UPLC method with a 1.8 min run time was employed for analysis. The mobile phase comprised of $0.1 \%$ formic acid $\mathrm{v} / \mathrm{v}$ in acetonitrile and $0.1 \%$ formic acid $\mathrm{v} / \mathrm{v}$ in water and the flow rate was $0.7 \mathrm{ml} / \mathrm{min}$. Separation was achieved using BEH C18column $(1.7 \mu \mathrm{m}, 2.1 \mathrm{~mm} \times 50 \mathrm{~mm}, 3 / \mathrm{pkg})$, Waters, USA) maintained at $45{ }^{\circ} \mathrm{C}$ employing an injection volume of $5 \mu \mathrm{l}$ for in vivo plasma samples.

\section{Optimisation of UPLC-MS/MS condition}

Final MRM transitions selected based on the signal to noise ratio $(\mathrm{S} / \mathrm{N})$ ratio with on-column injection analysis. Curtain gas, collision gas induced dissociation, ion spray voltage, and temperature set to $25.00 \mathrm{psi}, 6 \mathrm{psi}, 5500.00 \mathrm{v}$ and $550.00 \mathrm{C}$, respectively. Positive-ion electron spray ionisation mode was used, and MRM transitions of 823.8/151.2 for RIF and 266.2/234.0 for albendazole was monitored. 

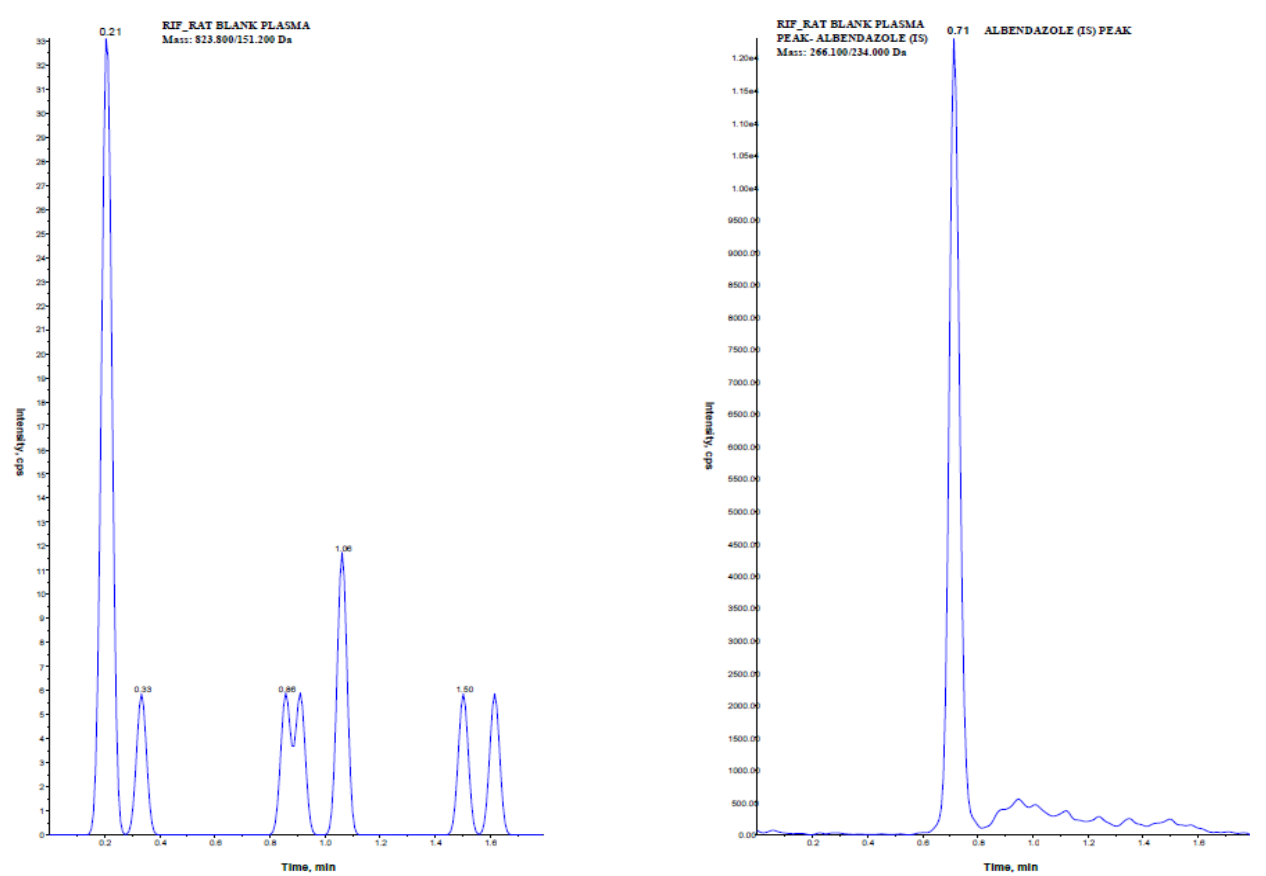

Fig.1: Representative chromatograms of rat blank plasma using RIF and albendazoleUPLC/MS/MS method
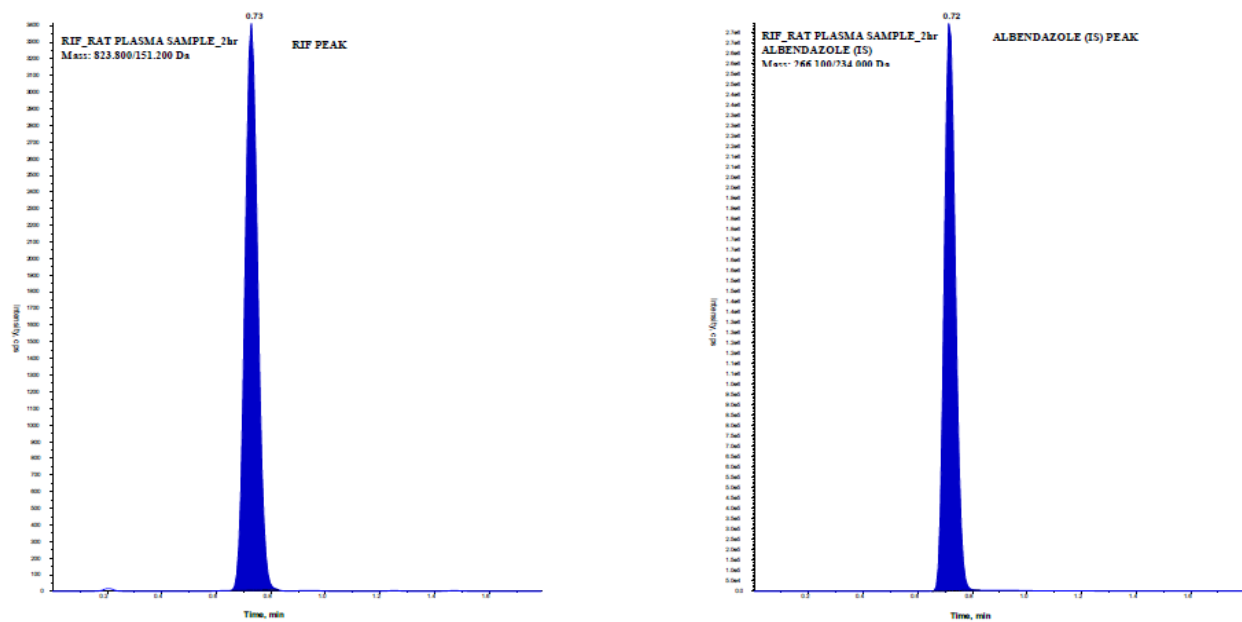

Fig.2: Representative chromatograms of RIF and albendazole in rat plasma sample

\section{Sample clean-up}

The next step was to develop the simple and efficient sample clean up devoid of matrix effect and interference from endogenous plasma components for estimation of RIF in rat plasma. Precipitation method was tried with liquid-liquid extraction (LLE) using ether and different combinations of hexane and ethyl acetate $(80-20 \%$, $\mathrm{v} / \mathrm{v}), \mathrm{n}$-hexane and isopropyl alcohol $(2-5 \%, \mathrm{v} / \mathrm{v})$ was also tried but none of these was found suitable to give good and consistent recovery for RIF. Finally, precipitation method with $100 \%$ acetonitrile tried and found suitable to give the best recovery for RIFand IS.For determination of matrix effect, drug-free control plasma extracted using the described method and drug added in extracted supernatant. Matrix effect determined by comparing the analytical response of these samples with that of standard solutions.

\section{Method validation}

Accuracy, precision, selectivity, sensitivity, linearity and stability were measured and used to assess the performance. UPLC-MS/MS analysis of the blank plasma samples showed no interference with the quantification of RIF and the IS (albendazole). Method specificity was established with pooled and individual plasma samples from six different sources. The retention times of RIF and the IS showed less variability with a percent coefficient of variance $(\% \mathrm{CV})$ well within acceptable limits of $5 \%$.

\section{LOD (limit of detection) and LOQ (limit of quantification)}

Two criteria were used to define LOQ, i.e., (1) the analytical response at LOQ must be five times the baseline noise and (2) the analytical response at LOQ can be detected with sufficient accuracy (80-120\%) and precision (20\%). LOD is defined as the lowest concentration of the analyte at which the signal is larger than three times the baseline noise. The measured LOQ and LOD values were 1.97 and 2 arbitrary units for RIF. The limit of quantification (LOQ) was set at $1.97 \mathrm{ng} / \mathrm{ml}$ These results well met the requirements of quantification of all analytes in plasma.

\section{Linearity}

The peak area ratios of the analyte to IS in rat plasma were linear over the concentration range 1.97 to $5047.00 \mathrm{ng} / \mathrm{ml}$ for RIF. The calibration model selected based on the analysis of the data by linear 
regression with and without intercepts $(y=m x+c$ and $y=m x)$ and weighting factors $\left(1 / x, 1 / x^{2}\right.$ and $\left.1 / \log x\right)$. The best fit for the calibration curve could be achieved by a linear equation of $y=m x+c$ and a $1 / \mathrm{x}^{2}$ weighting factor for all components. The correlation coefficients $\left(\mathrm{R}^{2}\right)$ for all components were above 0.9964 over the concentration range used.

\section{Precision and accuracy}

The intra-day precision (expressed by percent coefficient of variation i.e. CV\% of replicate analyzes) was estimated on the four quality control levels and the within-batch precision on the ten calibration standard levels. Table 1shows the results obtained for the within batch and between batch precision for RIF. The precision for all these analytes under investigation was not exceeded $15 \%$ at any of the concentrations studied and well met the requirements of validation.

\section{Recovery}

The extraction recovery of RIF from rat plasma was estimated at their low, medium and high QC levels. Plasma samples (in six replicates) containing RIF at QC concentration level was also spiked with internal standards. The results of absolute recoveries ranged from 74.26 to $101.73 \%$ are indicated in table 2 .

Table 1: Accuracy and precision from QC samples of RIF in rat plasma

\begin{tabular}{|c|c|c|c|}
\hline \multirow[t]{2}{*}{ Drug } & \multirow{2}{*}{$\begin{array}{l}\text { Spiked } \\
\text { concentration } \\
(\mathrm{ng} / \mathrm{ml})\end{array}$} & Intra-day & Inter-day \\
\hline & & $\begin{array}{l}\text { Mean measured concentration (ng/ml); \%mean } \\
\text { Accuracy; \%mean CV }\end{array}$ & $\begin{array}{l}\text { Mean measured concentration }(\mathrm{ng} / \mathrm{ml}) ; \% \text { mean } \\
\text { Accuracy; \%mean CV }\end{array}$ \\
\hline \multirow[t]{4}{*}{ RIF } & 1.97 & $2.01 ; 101.99 ; 4.78$ & $2.03 ; 102.80 ; 4.85$ \\
\hline & 5.91 & $5.81 ; 98.37 ; 8.61$ & $5.86 ; 99.12 ; 8.50$ \\
\hline & 2775.85 & $2860.93 ; 103.06 ; 8.47$ & $2831.32 ; 102.03 ; 8.24$ \\
\hline & 3785.25 & $3925.86 ; 103.71 ; 10.11$ & $3933.32 ; 103.91 ; 10.09$ \\
\hline
\end{tabular}

Data of $n=6$ replicates

Table 2: Extraction recovery of RIF in rat plasma

\begin{tabular}{lll}
\hline Analyte & Concentration $(\mathbf{n g} / \mathbf{m l})$ & \%mean recovery \\
\hline RIF & 5.91 & 74.26 \\
& 2775.85 & 82.77 \\
& 3785.25 & 101.73 \\
\hline
\end{tabular}

Data of $n=6$ replicates

\section{Stability}

QC samples subjected to short-term and long-term storage condition $\left(-70^{\circ} \mathrm{C}\right)$, freeze-thaw stability, auto-sampler stability and bench-top stability studies.All stability studies carried out at two concentration levels (low and high QC) in six replicates. The bench top stability was studied for low and high QC samples kept at room temperature (25
${ }^{\circ} \mathrm{C}$ ) for $6 \mathrm{~h}$. Freeze-thaw stability of low and high $\mathrm{QC}$ samples evaluated after 3 freeze-thaw cycles.The auto-sampler stability studied for low and high QC samples stored at autosampler at $10^{\circ} \mathrm{C}$ for $24 \mathrm{~h}$. The freezer storage stability of the drug in plasma determined by comparing the low and high QC samples stored for 30 $\mathrm{d}$ at $-70{ }^{\circ} \mathrm{C}$.The results indicated that each analyte had an acceptable stability under the conditions shown in table 3.

Table 3: Stability of RIF in rat plasma

\begin{tabular}{llllll}
\hline Drug & $\begin{array}{l}\text { Nominal } \\
\text { concentration (ng/ml) }\end{array}$ & Sample condition & & \\
\cline { 3 - 6 } & & $\begin{array}{l}\text { Bench top stability } \\
\text { \% accuracy; \%CV }\end{array}$ & $\begin{array}{l}\text { Auto-sampler stability } \\
\text { \% accuracy; \%CV }\end{array}$ & $\begin{array}{l}\text { Freeze-thaw stability } \\
\text { \% accuracy; \%CV }\end{array}$ & $\begin{array}{l}\text { 30 D. storage stability } \\
\text { \% accuracy; \%CV }\end{array}$ \\
\hline RIF & 5.91 & $97.58 ; 5.26$ & $99.79 ; 6.62$ & $98.49 ; 3.63$ & $101.52 ; 5.77$ \\
& 3785.25 & $104.92 ; 6.84$ & $102.87 ; 7.27$ & $103.10 ; 5.35$ & $96.44 ; 8.57$ \\
\hline
\end{tabular}

Data of $\mathrm{n}=6$ replicates; axposed at ambient temperature $\left(25^{\circ} \mathrm{C}\right)$ for $6 \mathrm{~h}$; ${ }^{\text {bept }}$ at an auto-sampler temperature $\left(10^{\circ} \mathrm{C}\right)$ for $24 \mathrm{~h}$; $\mathrm{c} A \mathrm{fter}$ three freezethaw cycles; dStored at $-70^{\circ} \mathrm{C}$.

\section{Sample dilution}

To demonstrate the ability to dilute and analyze samples containing RIF at the concentration above the upper limit of quantification, a set of plasma samples prepared to contain RIF at a concentration of $10094.00 \mathrm{ng} / \mathrm{ml}$, and placed in a $-70{ }^{\circ} \mathrm{C}$ freezer overnight till analysis. After thawing, the certain aliquot diluted either with 2 or 10 times withrat plasma and analyzed. The results of this experiment indicated that the dilution integrity of all the plasma samples was less than $15 \%$ of their respective nominal concentrations.

\section{Advantages of present method}

The aim of present investigation was to develop and validate a simpleUPLC-MS/MS method using gradient mode with sufficient accuracy and precision for RIF and its subsequent use in pharmacokinetic studies in rats. The present method involves simple precipitation procedure with good sensitivity and a gradient reverse-phase UPLC analysis for RIF.

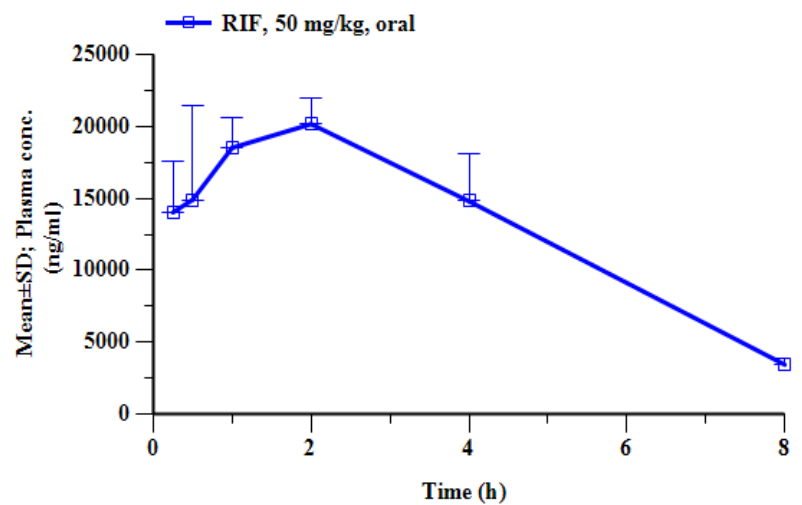

Fig.3: Mean plasma concentration vs. time following a single dose oral administration of RIF in male rats 
This method is specific for RIF with no interference and with good linearity, accuracy, and precision. This method involves only $25 \mu \mathrm{l}$ of plasma and in acetonitrile precipitation procedure; we achieved a high level of extraction efficiency for RIF. This makes the assay highly reproducible and allows us to lower the limit of quantification. Furthermore, this one-step precipitation extraction procedure uses simple, low cost and short run time. The chromatographic conditions of this method were optimized for a short 1.8 min run time in UPLC-MS/MS.

\section{Application to pharmacokinetic study}

The method described above was successfully applied to a pharmacokinetics evaluation of RIF. Following oral administration of RIF at $50 \mathrm{mg} / \mathrm{kg}$ oral suspension formulation male Wistar rats, plasma concentrations were quantifiable up to $8 \mathrm{hr}$ (last sampling point) with Tmax at $2.0 \mathrm{hr}$.The plasma concentration time profile of RIF shown in fig. 3 and mean pharmacokinetic parameters presented in table 4 .

Table 4: Mean pharmacokinetic parameters following single intravenous administration of RIF in male rats

\begin{tabular}{lllll}
\hline Analyte & $\mathbf{T}_{\max }(\mathbf{h})$ & $\mathbf{C}_{\max }(\mathbf{n g} / \mathbf{m l})$ & AUC $_{(\mathbf{0}-\mathrm{t})}\left(\mathbf{h}^{*} \mathbf{n g} / \mathbf{m l}\right)$ & AUC $_{(\mathbf{0}-\text { inf) }}\left(\mathbf{h}^{*} \mathbf{n g} / \mathbf{m l}\right)$ \\
\hline $\mathrm{RIF}$ & 1.67 & 20528.68 & 104498.83 & 125742.72 \\
\hline
\end{tabular}

Data of $n=3$ replicates; AUC: area under the concentration-time curve; $C_{\max }$ :peak plasma concentrations;

\section{CONCLUSION}

The UPLC-MS/MS bioanalytical method for determination of RIF was developed and validated in rat plasma. The method was sensitive enough to detect low concentration of $0.20 \mathrm{ng} / \mathrm{ml}$ for RIF. Recovery of RIF from spiked control samples were $>74 \%$ by using convenient and rapid precipitation method using acetonitrile. Intra and interday accuracy and precision of the validated method within the acceptable limits of $<20$ at LLOQ and $<15 \%$ at low, medium and high concentrations. The method was successfully applied to pharmacokinetics evaluation of RIF in rat plasma following oral administration.

\section{ACKNOWLEDGEMENT}

The authors are grateful to the management of UKA Tarsadia University, Bardoli, District Surat, Gujarat, India.

\section{CONFLICTS OF INTERESTS}

The authors declare no conflict of interest

\section{REFERENCES}

1. Fivy K, Syed ASS, Syed WG.Study on drug-resistant tuberculosis and tuberculosis treatment on patients with drug-resistant tuberculosis in chest clinic outpatient department.Int J Pharm Pharm Sci2012;4:733-7.

2. Minje H, Sun HJ, Jae HL, Kyoung, Junghan S, Sang HS. A method for simultaneous analysis of nine-second line antituberculosis drugs using UPLC-MS/MS. J Antimicrob Chemother 2013;68:2066-73

3. Thomayant P, Xiaoyan C, Christopher G, Donghui C, Ka LY,Jeanine B, et al. Drug-drug interaction studies: regulatory guidance and an industry perspective. AAPS J 2013;15:629-45.

4. Oesch F, Arand M, Strolin B, Castelli MD,Dostert P.Inducing properties of rifampicin and rifabutin for selected enzyme activities of the cytochrome P-450 and UDP-glucuronosyl transferase superfamilies in female rat liver. J Antimicrob Chemother 1996;37:1111-9.

5. Kathleen MG, Shiew-Mei H, Donald JT. Membrane transporters in drug development. Nat Rev Drug Discovery 2010;9:215-36.

6. Tomoki I, Tsuyoshi M, Koji A, Masakazu H, Noriko O, Takashi I. Integrated approach of in vivo and in vitro evaluation of the involvement of hepatic uptake organic anion transporters in the drug disposition in rats using rifampicin as an inhibitor. Drug Metab Dispos 2013;41:1442-9.

7. Guidance for Industry. Drug interaction studies-study design, data analysis, implications for dosing, and labeling recommendations draft guidance. US FDA; 2012. p. 1-79.

8. Sompon W, Kamonrat P, Virapong P. Cytochrome P450 enzyme mediated herbal drug interactions. EXCLI J 2014;13:869-96.

9. Sriram S, Prasanthi B, Tata S, Vijaya R. Development and validation of a high-performance liquid chromatographic method for the determination of rifampicin in human plasma. Int J PharmPharm Sci 2012;4:362-7.
10. Enoche FO. Spectrophotometric determination of isoniazid in pure and pharmaceutical formulations using vanillin. Int J Pharm Pharm Sci 2010;2:55-8.

11. Conte JE, Lin E, Zurlinden E. Liquid chromatographic determination of rifampicin in human plasma, bronchoalveolar lavage fluid, and alveolar cells. J Chromatogr Sci 2000;38:72-6.

12. McConnell JB, Smith H, Davis M, Williams R. Plasma rifampicin assay by an improved solvent extraction technique. Br JClin Pharmacol 1979;8:507.

13. Udupa N, Goyal P, Agarwal S, Venkatesh S, Pandey S. Simultaneous HPTLC determination of rifampicin and isoniazid in rat plasma. Indian J Pharm Sci 2003;65:414-6.

14. Calleja I, Blanco-Prieto MJ, Ruz N, Renedo MJ, Dios-Vieitez MC. High-performance liquid chromatographic determination of rifampicin in plasma and tissues. J Chromatogr A 2004;1031:289-94.

15. Khuhawar MY, Rind FMA. Liquid chromatographic determination of isoniazid, pyrazinamide and rifampicin from pharmaceutical preparations and blood. J Chromatogr B: Anal Technol Biomed Life Sci 2002;766:357-63.

16. Calleri E, De Lorenzi E, Furlanetto S, Massolini G, Caccialanza G. Validation of an RP-LC method for the simultaneous determination of isoniazid, pyrazinamide and rifampicin in a pharmaceutical formulation. J PharmaBiomed Anal 2002;29:1089-96.

17. Mohan B, Sharda N, Singh S. Evaluation of the recently reported USP gradient HPLC method for analysis of antituberculosis drugs for its ability to resolve degradation products of rifampicin. J PharmaBiomed Anal 2003;31:607-12.

18. Walubo A, Smith P, Folb PI.Comprehensive assay for pyrazinamide, rifampicin and isoniazid with its hydrazine metabolites in human plasma by liquid column chromatography. J Chromatogr B: Biomed Sci Appl 1994;658:391-6.

19. Woo J, Wong CL, Teoh R, Chan K. Liquid chromatographic assay for the simultaneous determination of pyrazinamide and rifampicin in serum samples from patients with tuberculosis meningitis. J Chromatogr B: Biomed Sci Appl 1987;420:73-80.

20. Ishii M, Ogata H. Determination of rifampicin and its main metabolites in human plasma by high-performance liquid chromatography. J Chromatogr B: Biomed Sci Appl 1988;426:412-6.

21. Seifart HI, Kruger PB, Parkin DP, Van JPP, Donald PR.Therapeutic monitoring of antituberculosis drugs by direct in-line extraction on a high-performance liquid chromatography system. J Chromatogr B: Biomed Sci Appl 1993;619:285-90.

22. Smith PJ, Dyk JV, Fredericks A. Determination of rifampicin, isoniazid and pyrazinamide by high-performance liquid chromatography after their simultaneous extraction from plasma.Int J Tuberculosis Lung Disease 1999;3:S325-8.

23. Balbao MS, Bertucci C, Bergamaschi MM, Queiroz RHC, Malfara WR, Dreossi SAC. Rifampicin determination in plasma by stir barsorptive extraction and liquid chromatography. J Chromatogr B: Biomed SciAppl 2010;5:1078-83. 
24. Gikas E, Bazoti FN, Fanourgiakis P, Perivolioti E, Roussidis A, Skoutelis A. Simultaneous quantification of daptomycin and rifampicin in plasma by ultra-performance liquid chromatography application to a pharmacokinetic study. JChromatogr B: Biomed Sci Appl 2010;51:901-6.

25. Hartkoorn RC, Khoo S, Back DJ, Tjia JF, Waitt CJ, Chaponda MA. Rapid and sensitive HPLC-MS method for the detection of plasma and cellular rifampicin.J Chromatogr B: Anal Technol Biomed Life Sci 2007;857:76-82.

26. Korfmacher WA, Bloom J, Churchwell MI, Getek TA, Hansen JEB, Holder CL. Characterization of three rifampicin via electrospray mass spectrometry and HPLC-thermospray mass spectrometry. J Chromatogr Sci 1993;31:498-501.
27. Baietto L, Avolio A, De Rosa FG, Garazzino S, Michelazzo M, Ventimiglia G. Development and validation of a simultaneous extraction procedure for HPLC-MS quantification of daptomycin, amikacin, gentamicin, and rifampicin in human plasma. Anal Bioanal Chem 2010;396:791-8.

\section{How to cite this article}

- Aslam Burhan, Bhavin Vyas. A rapid, sensitive and validated ultra performance liquid chromatography and tandem mass spectrometry method for determination of rifampicin in rat plasma: application to pharmacokinetic study.Int J Pharm Pharm Sci 2017;9(2):222-227. 